\begin{tabular}{|c|l|}
\hline Title & Emoticon Recommendation System to Richen Y our Online Communication \\
\hline Author(s) & U rabe, Y uki; Rzepka, Rafal; A raki, Kenji \\
\hline Citation & $\begin{array}{l}\text { International Journal of Multimedia Data Engineering and Management, 5(1), 14.33 } \\
\text { https://loi.org/10.4018/jmdem.2014010102 }\end{array}$ \\
\hline Issue Date & 2014 \\
\hline Doc URL & http://hdl.handle.net/2115/63958 \\
\hline Type & article (author version) \\
\hline File Information & Emoticon-Recommendation-System-to-Richen-Your-Online-Communication.pdf \\
\hline
\end{tabular}

Instructions for use 


\title{
Emoticon Recommendation System to Richen Your Online Communication
}

\author{
Yuki Urabe, Graduate School of Information Science and Technology, Hokkaido University, \\ Sapporo, Japan
}

Rafal Rzepka, Graduate School of Information Science and Technology, Hokkaido University, Sapporo, Japan

Kenji Araki, Graduate School of Information Science and Technology, Hokkaido University, Sapporo, Japan

\begin{abstract}
Japanese emoticons are widely used to express users'feelings and intentions in social media, blogs and instant messages. Japanese smartphone keypads have a feature that shows a list of emoticons, enabling users to insert emoticons simply by touching them. However, this list of emoticons contains more than 200, which is difficult to choose from, so a method to reorder the list and recommend appropriate emoticons to users is necessary. This paper proposes an emoticon recommendation method based on the emotive statements of users and their past selections of emoticons. The system is comprised of an affect analysis system and an original emoticon database: a table of 59 emoticons numerically categorized by 10 emotion types. The authors' experiments showed that $73.0 \%$ of chosen emoticons were among the top five recommended by the system, which is an improvement of $43.5 \%$ over the method used in current smartphones, which is based only on users' past emoticon selections.
\end{abstract}

Keywords: $\quad$ Affect Analysis, Application, Emoticon, Recommendation System, Smartphone

\section{INTRODUCTION}

CMC (Computer-Mediated Communication) has become popular in recent years as it allows people to communicate regardless of time, limitations of physical distance, and familiarity (i.e. whether or not they know each other). However, in contrast to F2F (Face-to-Face) communication, text-based communication in CMC lacks the ability to convey nonverbal cues such as facial expression, attitude, and tone of voice (Jibril \& Abdulah, 2013). These cues take up an estimated $93 \%$ of every day communication (Mehrabian, 1971) and enable humans to understand others' feelings and intentions not only from the spoken words but also from 
their facial expressions showing emotion and attitudes (Fridlund, 1994). Therefore, we need to find a way to compensate for this lack of nonverbal cues in order to prevent confusion and express user intentions fully in CMC.

Emoticons, marks expressing faces or movement composed of letters and symbols, may serve as nonverbal surrogates in CMC (Walther \& D'addario, 2001; Ptaszynski, 2012; Wei, 2012). Emoticons are used in CMC to express one's feelings, enhance the sentence, and express humor (Derks, Bos \& Grumbkow, 2008). Recipients can understand the sender's intended emotions, attitudes, and attention clearly with emoticons in the sentence rather than by receiving only words in the sentence (Lo, 2008; Gajadhar \& Green, 2005; Ip, 2002). Information conveyed by emoticons has a great importance in $\mathrm{CMC}$ which we should not ignore, and thus, research on emotion analysis from emoticons, and development of interfaces which support users expressing their feelings using emoticons are highly important.

Emoticons can be divided into two types: vertical style (e.g. ":)"), mainly used in western countries and horizontal style (e.g. " $\left(\wedge \_\wedge\right)$ "), mainly used in Asian countries (Park, Barash, Fink \& Cha, 2013). The vertical style emoticon is composed of symbols and English alphabet and is rotated by 90 degrees. Contrary to the vertical one, the horizontal style emoticon is un-rotated and easily comprehensible to a reader (Park et al., 2013). Moreover, the horizontal style is composed of symbols and many different kinds of characters from languages such as Japanese, Korean, Cyrillic, and so on (Robb, 2013; Pollack, 1996; Ashcraft, 2012). The number of emoticons in both styles differs greatly; that is, there are around 260 vertical style emoticons whereas horizontal style emoticons exceed 58,000 and are still increasing in recent years. These large numbers of emoticons are sophisticated enough to express nuances in meaning and may richen the quality of communication in CMC. However, it is difficult for users to find appropriate emoticons to express their intentions from these 58,000 emoticons.
An emoticon dictionary also appears along with keypads in smartphones such as the iPhone from the iOS 4.2.1 to the latest ("^ ^^" button in Japanese and Chinese keypads) (Dilger, 2010). The number of emoticons in the dictionary in the Japanese and Chinese iPhone keypads are 226 and 265, respectively. These numbers are still high for users to choose from, hence a method to assist users in choosing appropriate emoticons that match their input is necessary. In the Japanese iPhone keypad, we have discovered that emoticons are recommended from users' past selection of emoticons. However, emoticons are chosen depending on the valence of the input, i.e., positive emoticons are likely to be chosen in positive contexts, and vice versa (Kato, Kato \& Scott, 2009; Ip, 2002). Therefore, we hypothesize that recommending emoticons according to the emotion type from users' input will support users in finding appropriate emoticons for their statements more easily. Also, the choice of emoticons varies for each individual (Tossell, Kortum, Shepard, Barg-Walkow, Rahmati, \& Zhong, 2012; Wolf, 2000), hence we assume that incorporating a function that recommends emoticons according to users' preferences would be more helpful for users. In this paper, we propose three types of emoticon recommendation system that help users to easily find and insert appropriate emoticons for their input. We will also examine which of these three systems performs the best, and compare our system with the current method used in the Japanese iPhone keypad.

Our emoticon recommendation systems are comprised of an original emoticon database, a table of emoticons organized according to the gradation of 10 distinct emotions. In order to analyze users' intended emotion from the emotional expressions used in the sentence, we employed an affect analysis system called ML-Ask (Ptaszynski, Dybala, Rzepka \& Araki, 2008). The difference between these systems is the method of reordering the emoticon database according to the output given by ML-Ask.

The outline of this paper is as follows: in the next section, we present related research and 
describe some differences between several studies. The section after describes the procedure of our emoticon recommendation system, detailed information about ML-Ask and the originally created emoticon database, and three types of emoticon recommending system we developed by changing the method for optimizing emoticons in the database. The section after that details an evaluation experiment investigating which of the proposed three systems perform the best and how they perform differently to the current emoticon recommending system used in smartphones. Finally, conclusions and future works are presented in the last section.

\section{RELATED WORKS}

In the field of artificial intelligence, an automatic emoticon analysis system, "CAO" was developed by Ptaszynski, Maciejewski, Dybala, Rzepka and Araki (2010) based on the theory of kinesics. "CAO" extracts an emoticon from the input and determines the specific emotion type. The system's coverage exceeds three million possibilities. Moreover, several tests proved that $99.5 \%$ of emoticons were correctly deduced and that $85.2 \%$ of the emotions in the emoticons were correctly estimated. Additionally, an emoticon recommendation method based on the estimation of emotions, communication, and action types written by users was proposed by Emura and Seki (2012). The results of this system showed that by recommending emoticons not only from emotion categories but also from communication and action types, $66.7 \%$ of the suggested emoticons were suitable; this was a significant improvement over the recommendation results that only utilized the emotion categories. The emoticons in CAO's emoticon database and emoticon recommendation system by Emura and Seki (2012) were both simply categorized according to such emotion types. Meanwhile, a normative table of emotions and emphasis of emoticons was developed by Kawakami (2008), by conducting a questionnaire to university students. The focus was different from the aforementioned two studies in that Kawakami concentrated on how much an emoticon expresses each emotion and investigated how much the emoticon emphasizes the sentence. The research analyzed 31 emoticons and found that some emoticons strongly express more than one emotion.

The idea of the normative table of emotions expressed by emoticons was helpful in developing a more accurate emoticon recommendation system. Creating a database of emoticons showing a numerical expression of each emotion could be a step toward the creation of a system that can recommend emoticons that express the user's complicated emotional state.

\section{EMOTICON RECOMMENDATION SYSTEM}

Our proposed emoticon recommendation system basically performs by utilizing two main procedures: the affect analysis system ML-Ask (Ptaszynski et al., 2008), and an originally created emoticon database. The system process is as follows (see Figure 1):

1. A user inputs a sentence that includes an emotive word;

2. ML-Ask examines the input and outputs the emotion type;

3. The emoticon database reorders the database according to the emotion type output by ML-Ask and shows a row of emoticons to the user;

4. The user chooses the appropriate emoticon from the list and the system automatically inserts the emoticon directly after the input.

\section{Affect Analysis System: ML-Ask}

Ptaszynski et al. (2008) created an affect analysis system, ML-Ask, for Japanese utterances. MLAsk firstly separates emotive words from nonemotive words, and searches for expressions of specific emotion types in the emotive words. The expressions are based on a Japanese emotive word dictionary created by Nakamura (1993), in which he defines emotions into 10 different 


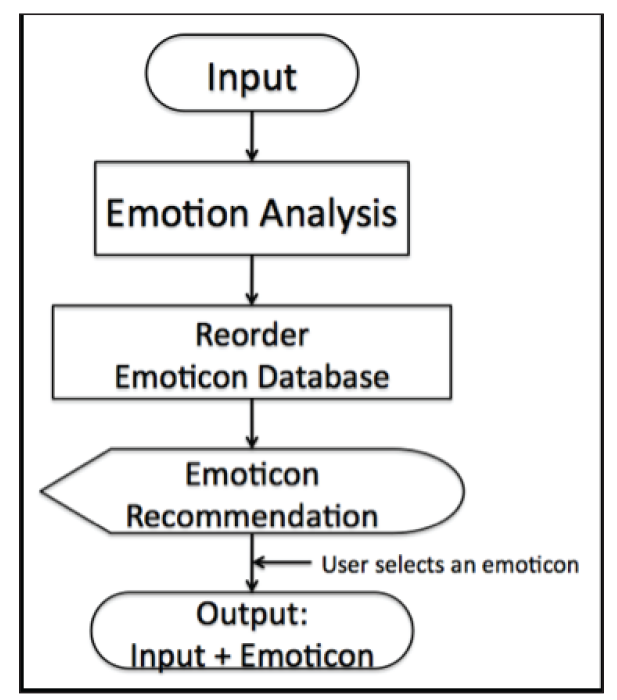

types: joy/delight, anger, sadness/gloom, fear, shyness, fondness/liking, dislike, excitement, relief, and surprise/amazement. We employed ML-Ask in our system because our emoticon database also applied Nakamura's definition of ten emotion types and is completely compatible with ML-Ask.

Figure 2 is an example of output from ML-Ask. It generates output as follows: input, the number of emotions: number, the type of emotion: emotive word, two dimension (positive or negative and active or passive), whether the input expresses as positive or negative, and whether the input is active or passive. We only employed "the type of emotion" (the underlined part in Figure 2) in order to reorder the emoticon database.

\section{Emoticon Database}

We manually constructed an emoticon database by conducting a questionnaire towards 60 Japanese university students. The purpose of the questionnaire was to investigate the power of each of 10 emotions expressed by an emoticon, in order to acquire human perceptions towards emoticons. The participants rated 59 chosen emoticons from iOS 5.0 by grading the extent to which each emoticon expressed each of the ten emotion types on a 5-point scale. We calculated the average values of the participants' ratings and linked the values to each emoticon to create the database. The condition for the chosen emoticons was those with no symbols outside of brackets (i.e. " $\left({ }^{\wedge} 0^{\wedge}\right)$ " qualifies, but " $\backslash\left({ }^{\wedge} 0^{\wedge}\right) /{ }^{\prime} "$ is excluded. In these emoticons, “^” and " 0 " represent the eyes and mouth of a face, respectively. Also, the two slashes outside the brackets symbolize the arms of a human body.) We focused only on emoticons that express facial expressions and have no symbols outside of the brackets in order to discover what kind of emoticons users choose based on the differences in the facial expressions. After we find a pattern for which emoticons express which emotion types for emoticons without symbols outside of brackets, we will expand the database by also including emoticons that have symbols outside of brackets.

The reason why we chose to conduct a questionnaire to create an emoticon database rather than collecting data automatically from social media is that emoticons are mostly used to help people to interpret the nuance of the sentence and the level of emotion not captured by language elements alone (Lo, 2008). Moreover, most of the words in sentences that have emoticons do not use emotional expressions, so it is difficult to analyze the emotion from the sentence and investigate which emotion is expressed by the emoticon. The emoticon 
Figure 2. Example of output from ML-Ask

今日は楽しかった|emotions: (1)|YOR:楽しい||2D|POS|ACT_or_PAS
<Translation>
Today was fun lemotions: (1)|YOR: fun ||2D|POS|ACT_or_PAS
YOR: Joy/Delight, POS: Positive, ACT: Active, PAS: Passive

analysis system CAO (Ptaszynski et al., 2010) is capable of analyzing the emotion type of the emoticon, but does not calculate how strongly the emoticon expresses the emotion. Therefore, we conducted a questionnaire to collect perceptions of the power of the emotion expressed in each emoticon:

- Survey: Sixty university students participated in the questionnaire in July 2012. The participants consisted of 22 men and 38 women, and the average of their ages was $20.71(\mathrm{SD}=1.21)$. The participants rated 59 emoticons by grading the extent to which each emoticon expressed each of the ten emotion types on a 5-point scale. We phrased the question as follows: "On a 5-point scale, please rate how well the emoticon expresses each of the ten emotions. (1. Not expressed 2. Poorly expressed 3. Neutral 4. Somewhat expressed 5. Expressed). We also demonstrated an example of rating (Figure 3 ) and indicated words that embody each emotion;
- Results: Figure 4 is an example of how strongly an emoticon expresses each of the ten emotions (Figure 4; minimum is 1.00 , maximum is 5.00 ). The average rating values of each emotion for the 59 emoticons were calculated by collecting the participants' ratings and using those values to create a database (Urabe, 2013). In our systems, we use this database to rearrange emoticons in order of the emotion type analyzed by ML-Ask and recommend emoticons from the top.

From the participants' ratings, we discovered that 35 out of 59 emoticons scored more than 3.00 in at least two emotion types, which means that those emoticons indicate plural emotion types. For example, the emoticon "(*^ $\bigcirc \wedge *)$ " scored 4.85 points in "joy/delight", 4.24 points in "fondness/liking", and 3.55 points in "relief" while the other seven emotion types were below 2.00 points (Figure 4). Moreover, we discovered two rules for emoticons that contain plural emotion types:

Figure 3. Example of emoticon ratings in each of the 10 emotions

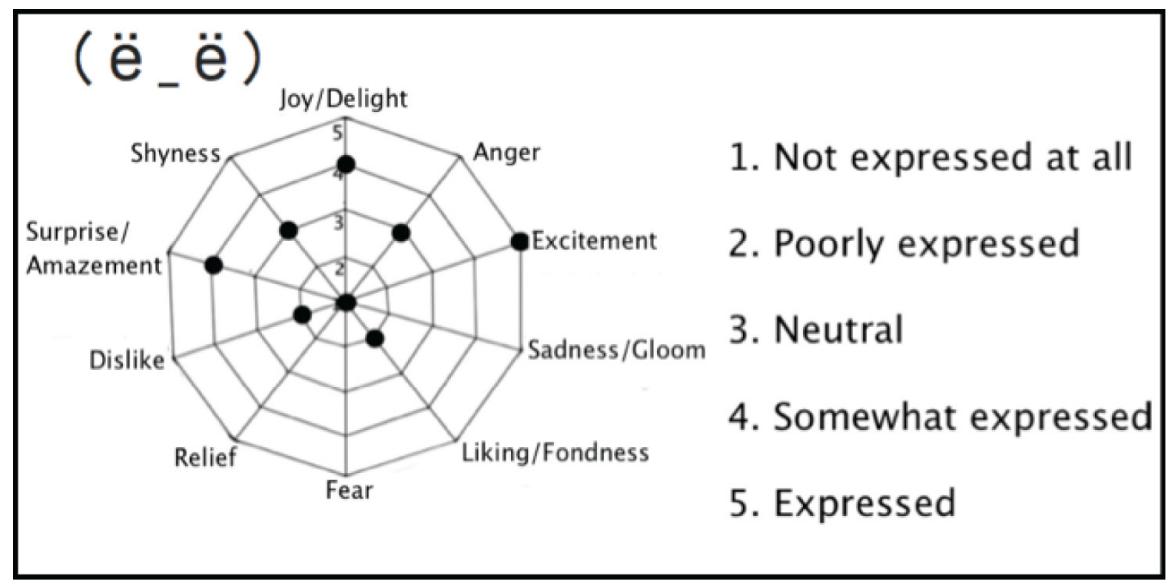




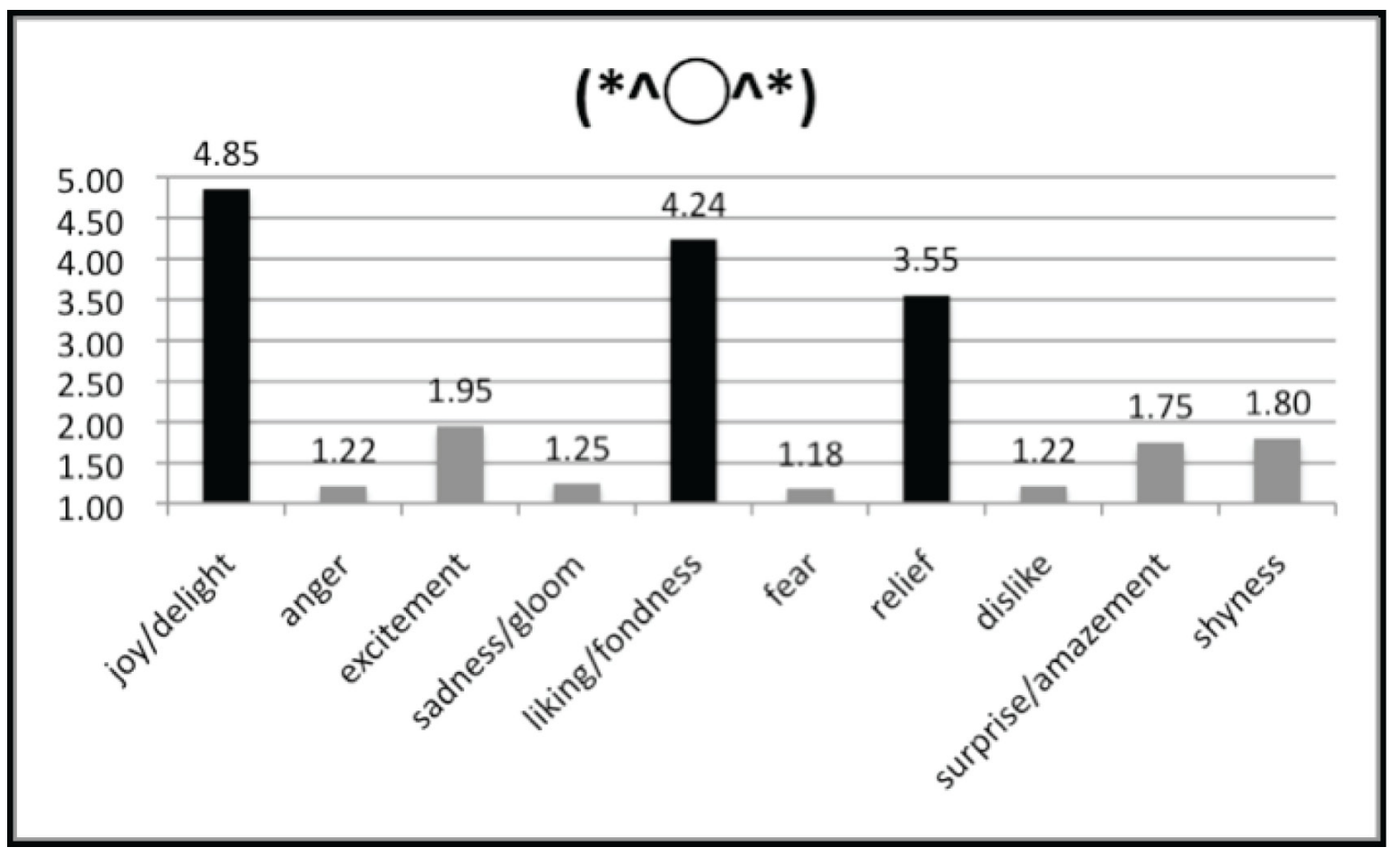

1. Emoticons that scored high for "joy/ delight" tend to score high for "fondness/ liking" and "relief" at the same time and receive low points of "anger", "sadness", "fear" and "dislike". Other emoticons expressed in this way had the same pattern as Figure 4;

2. When emoticons scored highly for "sadness/gloom", they often also score highly for "dislike" and "fear" and receive low points for "fondness/liking", "joy/delight", and "relief".

These characteristics indicate that some emotion types are polarized.

We also computed the correlation coefficient and determined the closeness of emotion types using the 59 emoticons (Table 1). From Table 1, three emotion types, "joy/delight", "fondness/liking", and "relief" scored extremely high positive correlations with each other $(r=$ 0.989; "joy/delight" and "fondness/liking", $r=$ 0.973; "joy/delight" and "relief", and $r=0.968$; "fondness/liking" and "relief"). In contrast, three emotion types, "dislike", "fear" and "sad- ness/gloom" scored high positive correlations ( $r=0.645$; "dislike" and "sadness/gloom", $r$ $=0.796$; "dislike" and "fear", and $r=0.867$; "sadness/gloom" and "fear") with each other and negative correlations with "joy/delight", "fondness/liking", and "relief". Therefore, we can determine that three emotion types ("joy/ delight", "fondness/liking", and "relief") and four emotion types ("dislike", "fear", and "sadness/gloom") are polarized from each other. "Anger" showed a higher positive correlation with "dislike" and negative correlation with "joy/delight", "fondness/liking", and "relief". Although "anger" is thought to be negative emotion, it can be positioned somewhat differently to "fear" and "sadness/gloom". "Excitement" showed a positive correlation with "dislike", "shyness", and "surprise/amazement" and negative correlations with three positive emotion types. "Excitement" appears to lie close to negative emotion types from these results; however, we believe that this is due to the unbalanced number of emoticons expressing each emotion type in the database (Figure 5). The numbers of emoticons that express positive 
Table 1. Correlation coefficient of ten emotion types

\begin{tabular}{|c|c|c|c|c|c|c|c|c|c|c|}
\hline Emotion & 1 & 2 & 3 & 4 & 5 & 6 & 7 & 8 & 9 & 10 \\
\hline 1. joy/delight & & $-0.540^{\text {के }}$ & $-0.694^{\frac{1}{2}}$ & $-0.641^{*}$ & $0.989^{\frac{*}{*}}$ & $-0.825^{* 2 \pi}$ & $0.973^{\text {** }}$ & -0.900 & $-0.515^{* *}$ & ${ }^{*}-0.615^{*}$ \\
\hline 2. anger & $-0.540^{*-k}$ & & $0.560^{* *}$ & 0.169 & $-0.528^{* k}$ & 0.239 & $-0.548^{*}$ & $0.686^{\text {*k }}$ & 0.034 & $0.234^{*}$ \\
\hline 3. excitement & $-0.694^{*}$ & $0.560^{\text {** }}$ & & 0.256 & -0.701 & $0.594^{\frac{k+4}{4}}$ & -0.754 & 0.700 & $0.623^{*}$ & 0.728 \\
\hline 4. sadness/gloom & $-0.641^{*}$ & 0.169 & 0.256 & & -0.641 & $0.867^{\text {*t* }}$ & -0.643 & 0.645 & 0.059 & 0.391 \\
\hline 5. liking/fondness & $0.989^{* 2 \pi}-$ & $-0.528^{*}$ & -0.701 & -0.641 & & $-0.830^{*}$ & $0.968^{*}$ & -0.893 & -0.527 & -0.595 \\
\hline 6. fear & $-0.825^{k *}$ & 0.239 & $0.594^{\text {k* }}$ & $0.867^{* \pi / k}$ & $-0.830^{*}$ & & $-0.842^{*}$ & $0.796^{\text {k* }}$ & 0.463 & 0.651 \\
\hline 7. relief & $0.973^{* \pi}$ & $-0.548^{*}$ & -0.754 & -0.643 & $0.968^{*}$ & $-0.842^{*}$ & & -0.884 & -0.542 & -0.640 \\
\hline 8. dislike & -0.900 & $0.686^{\text {t*k }}$ & 0.700 & 0.645 & -0.893 & $0.796^{* 3 \pi}$ & -0.884 & & $0.351^{*}$ & 0.643 \\
\hline 9. surprise/amazement & $-0.515^{* *}$ & 0.034 & $0.623^{*}$ & 0.059 & -0.527 & 0.463 & -0.542 & $0.351^{*}$ & & 0.459 \\
\hline 10. shyness & $-0.615^{*}$ & $0.234^{*}$ & 0.728 & 0.391 & -0.595 & $0.651^{2 \pi}$ & -0.640 & 0.643 & 0.459 & \\
\hline
\end{tabular}

* shows a significance level less than 0.05

** shows a level less than 0.01

Figure 5. Number of emoticons rated more than 3.0 for each emotion type

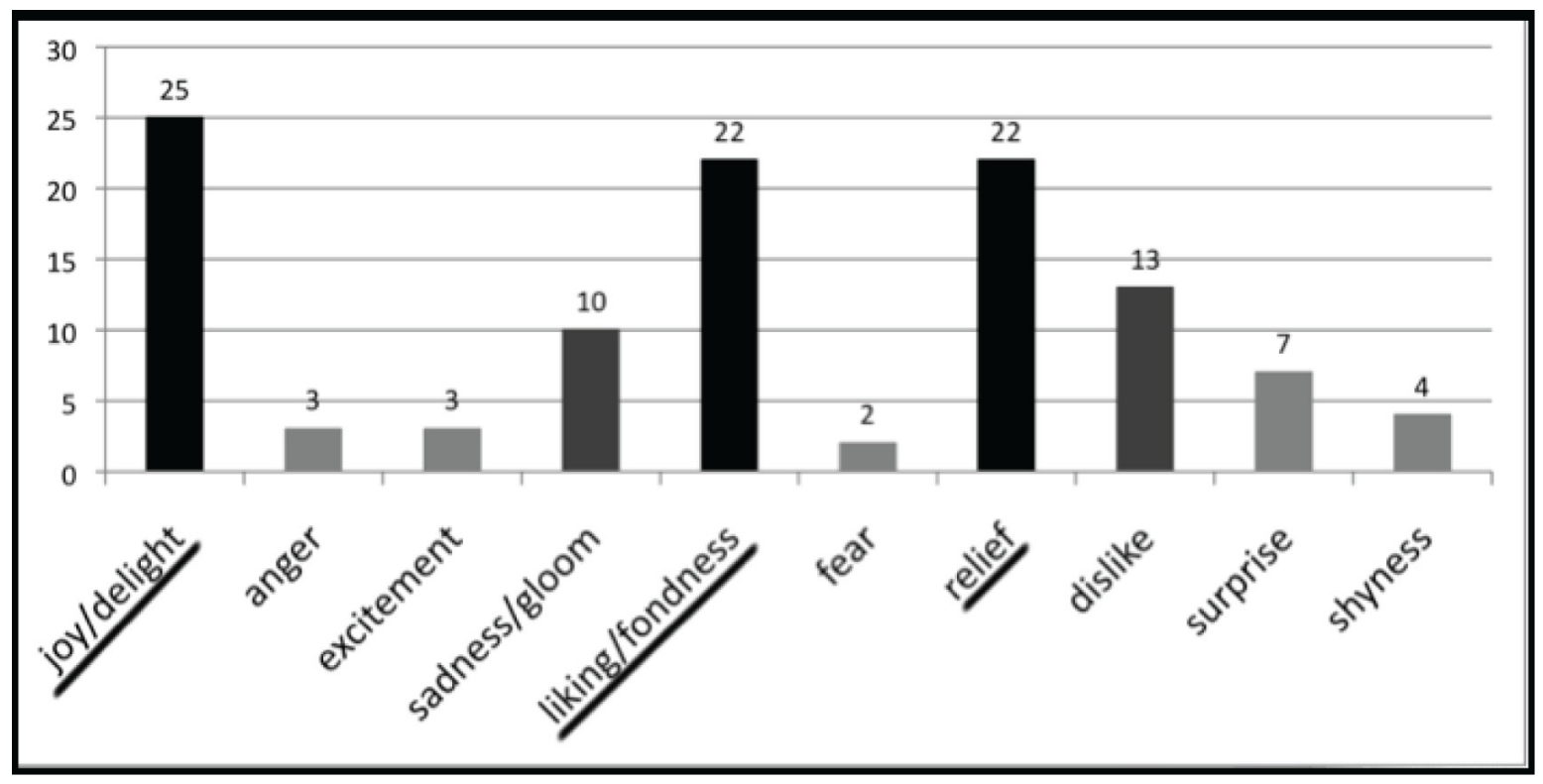

(“joy/delight, fondness/liking", and "relief”) facial expressions are significantly higher than negative ("sadness/gloom", "fear", "dislike", and "anger") orneutral("excitement", "surprise/ amazement", and "shyness") ones. Therefore, it is necessary to expand and balance out the number of emoticons expressing each emotion type in the database.
We developed three systems by integrating ML-Ask and the emoticon database. The first system is an emotion-based emoticon recommendation system called "EBReS", which recommends emoticons based only on users' emotional statements. The other two systems recommend emoticons based on users' emotional statements and users' past emoticon 
selections. The difference between these two systems is which aspect we prioritized; users' past emoticon selection or the emotional input. One of these two systems is called "HiBReS- $\varepsilon$ ", which recommends emoticons prioritizing users' past selection of emoticons over the emotion type from the input. The other is called "EBReS- $\eta$ " which prioritizes the emotion type from the input over users' past selection of emoticons. Further details about these systems are described below.

\section{HiBReS (History-Based Emoticon Recommendation System)}

The method employed in this system is currently used in the Japanese iOS keypad when recommending emoticons to users. We will refer to this as "HiBReS" in this paper. The characteristic of this system is that the system recommends emoticons based on users' past emoticon selection. For example, when a user chooses emoticon A first, emoticon A will be at the top of the recommendations when choosing emoticons for the second time. This method is relatively useful for users who only use a limited number of emoticons. However, this is not a suitable system for users who prefer to use various kinds of emoticons.

\section{EBReS (Emotion-Based Emoticon Recommendation System)}

The EBReS (Emotion-based emoticon recommendation system) recommends emoticons to users by reordering the database based on their emotional statements. According to a study by Kato et al. (2009), positive emoticons are used more often in positive contexts, and vice versa, therefore this system is helpful not only for users who tend to use various kinds of emoticons, but also for users who are unfamiliar with emoticons. However, this system does not consider users' past selections, so users who use only a limited number of emoticons may find it less helpful.

\section{HiBReS- $\varepsilon$ (History-Prioritized Emotion Based Emoticon Recommendation System)}

The HiBReS- $\varepsilon$ (History-prioritized emotionbased emoticon recommendation system) firstly recommends emoticons based on users' past emoticon selections, regardless of emotion types, and recommends the remaining emoticons to users based on users' emotional input. This system has the advantage of recommending emoticons that users often choose, and when they want to choose other emoticons, these are placed right after those emoticons.

\section{EBReS-n (Emotion-Prioritized History-Based Emoticon Recommendation System)}

The EBReS- $\eta$ (Emotion-prioritized historybased emoticon recommendation system) helps users to acquire appropriate emoticons by recommendations based on users' emotional statements. Moreover, the EBReS- $\eta$ especially recommends emoticons which users often choose based on their past selections when choosing emoticons to use with statements of the same emotion types in the past. This system firstly gathers emoticons that express similar emotions based on the input and especially considers users' emoticon preferences, so we anticipate that it may be the most user-friendly system among the four systems.

\section{Evaluation Experiment}

We developed a smartphone application in order to conduct an evaluation experiment towards users to confirm which of the aforementioned four systems is the most useful and recommends the most appropriate emoticons to users. Our application is usable on the iPhone (from iOS 7.0 to iOS 7.0.4). The device we used for the experiment was the iPhone 5S (iOS 7.0.4) due to its compatibility with the latest iOS at the time of writing. The experiment was performed 
over eight days from 31 st Oct 2013 to 8 th Nov 2013 with the cooperation of 30 Japanese undergraduate and master's students.

The experiment employed the semantic differential (SD) scale for the evaluation of the systems. We also examined what kind of emoticons users chose and the ranking of the recommendations from each system.

\section{Semantic Differential Scale}

Osgood, Suci and Tannenbaum (1957) created the SD scale, which is frequently used to investigate users' perception of an object (e.g. a system, place, etc.). In this method, the subject's perception of each system is quantified on a 7-point scale. We used 22 image-word pairs for the experiment (Table 2).

\section{Participants}

Thirty undergraduates and master's students participated in the experiment. The group consisted of 15 men and 15 womenn (Table 3 ). Their average age was 22.40 years old (SD $=1.77$ ). Among the 30 participants, $60.0 \%$ of the students possessed an iPhone or iPad, $33.3 \%$ possessed an Android device, and the rest possessed feature phones. Moreover, $86.7 \%$ of the students reported that they very often or somewhat often send emails daily, and $90.0 \%$ use emoticons very often or somewhat often when sending email.

\section{Device}

We used the iPhone 5S (iOS 7.0.4) for the experiment as our application is only usable in
iOS 7, and the iPhone 5S is the most compatible device with iOS 7. The application performs as follows:

1. When the user touches the input area, a Japanese keypad appears (Figure 6, 1 \& 2);

2. The user inputs a sentence (Figure 6, 3). In Figure 6,3, we entered “今日は楽しかつ た" (kyo ha tanoshikatta, meaning "I had a fun day today" in Japanese);

3. When the user touches the “顔文字” (kaomoji, meaning "emoticon" in Japanese) button, a list of emoticons appears (Figure $6,4)$;

4. The users selects an emoticon by scrolling up and down the list, and touches “決定” (kettei, meaning "done" in Japanese) button to confirm (Figure 6, 5);

5. The system automatically inserts the chosen emoticon right after the input (Figure 6,6).

\section{Procedure}

The procedure of the experiment was as follows:

1. Respondents firstly filled out basic information: their university year group, sex, age, faculty, whether they possess a smartphone, and whether they use emoticons when sending messages daily;

2. Respondents tested one of the four methods; the proposed three methods and the current method. The order in which a participant tested the four systems was decided by random selection in order to examine the difference between participants using each of the systems at the beginning;

\section{Table 2. 22 image-word pairs}

22 Image-Word Pairs (Translated from Japanese Used in Experiment)

Boring - Fun, Not impressive - Impressive, Unfriendly - Friendly

Difficult to use - Easy to use, Slow - Fast, Inconvenient - Convenient,

Unnecessary - Necessary, Heavy - Light, Obscure - Clear, Dislike - Like

Old - New, Complicated - Simple, Not interested - Interested, Common - Noble

Inaccurate - Accurate, Useless - Useful, Difficult to see - Easy to see

Difficult - Easy, Difficult to choose - Easy to choose, Ordinary - Special

Dumb - Smart, Unsatisfied - Satisfied 
Table 3. Demographic factors of participants

\begin{tabular}{|l|l|l|l|l|}
\hline \multicolumn{5}{|c|}{ Demographic Factors (n= 30) } \\
\hline \multicolumn{2}{|c|}{ Measure } & \multicolumn{1}{|c|}{$\begin{array}{c}\text { Men } \\
(\mathbf{n}=\mathbf{1 5})\end{array}$} & \multicolumn{1}{|c|}{$\begin{array}{c}\text { Women } \\
(\mathbf{n}=\mathbf{1 5})\end{array}$} & \multicolumn{1}{c|}{$\begin{array}{c}\text { Total } \\
(\mathbf{n}=\mathbf{3 0})\end{array}$} \\
\hline \multirow{3}{*}{ Device used daily } & iPhone/iPad & $6(20.0 \%)$ & $12(40.0 \%)$ & $18(60.0 \%)$ \\
& Android & $7(23.3 \%)$ & $3(10.0 \%)$ & $10(33.3 \%)$ \\
& Others & $2(6.7 \%)$ & $0(0.0 \%)$ & $2(6.7 \%)$ \\
\hline \multirow{5}{*}{ Daily email usage } & Very Often & $5(16.7 \%)$ & $11(36.7 \%)$ & $16(53.3 \%)$ \\
& Somewhat & $7(23.3 \%)$ & $3(10.0 \%)$ & $10(33.3 \%)$ \\
& Hardly & $3(10.0 \%)$ & $1(3.3 \%)$ & $4(13.3 \%)$ \\
& Not at all & $0(0.0 \%)$ & $0(0.0 \%)$ & $0(0.0 \%)$ \\
\hline \multirow{5}{*}{ Daily emoticon usage } & Very often & $4(13.3 \%)$ & $9(30.0 \%)$ & $13(43.3 \%)$ \\
& Somewhat & $8(26.7 \%)$ & $6(20.0 \%)$ & $14(46.7 \%)$ \\
& Hardly & $3(10.0 \%)$ & $0(0.0 \%)$ & $3(10.0 \%)$ \\
& Not at all & $0(0.0 \%)$ & $0(0.0 \%)$ & $0(0.0 \%)$ \\
\hline
\end{tabular}

3. Respondents rated the system using 22 image-word pairs on a 7-point scale;

4. Respondents tested the other three systems as written above in Steps 2 and 3.

We showed participants a list of 15 sentences that each included one emotive word, and asked them to enter the sentences in each of the four systems. The 15 sentences were comprised of five sentences with positive emotions (one of the emotions from joy/delight, relief, and liking/fondness), five neutral (one of the emotions from surprise/amazement, excitement, and shyness), and five negative (one of the emotions from sadness/gloom, anger, fear, and dislike) (examples shown in Table 4). The emotions used in the list were chosen randomly from the positive, negative, and neutral emotions. The sentences used in the list were chosen from participants' inputs from a previous experiment (Urabe, Rzepka \& Araki, 2013). Moreover, we performed a preliminary experiment to examine whether the chosen sentences express one of the 10 emotion types strongly by asking 10 Japanese people to rate them on a 5-point scale (minimum is 1.0 , maximum is 5.0 ; average points collected from respondents are written after the emotion types in Table 4).

\section{RESULTS AND DISCUSSION}

We investigated which of the four systems performed the best for users by analyzing them from four aspects: (a) the average rank of the emoticons chosen by users with the system; (b) the proportion of the emoticons chosen by users that were among the recommended top five; (c) the kind of emoticons users chose with the system; (d) the users'impressions of the system.

\section{Average Rank of Emoticons Chosen by Users with Each System}

We examined the average rank of the emoticons chosen by users with each system by calculating the average number of the emoticon rank. Figure 7 shows the overall result. As our aim of this research is to recommend appropriate emoticons to users that match the sentence, we can judge that the lower the value in this table, the better the system performs for users.

According to the overall result shown in Figure 7, the EBReS- $\eta$ appears to have the lowest value among the four systems at 5.0 points. Therefore, we can determine that the EBReS- $\eta$ recommends the most appropriate 
Figure 6. Application procedure (Device: iPhone 5S, iOS 7.0.4)

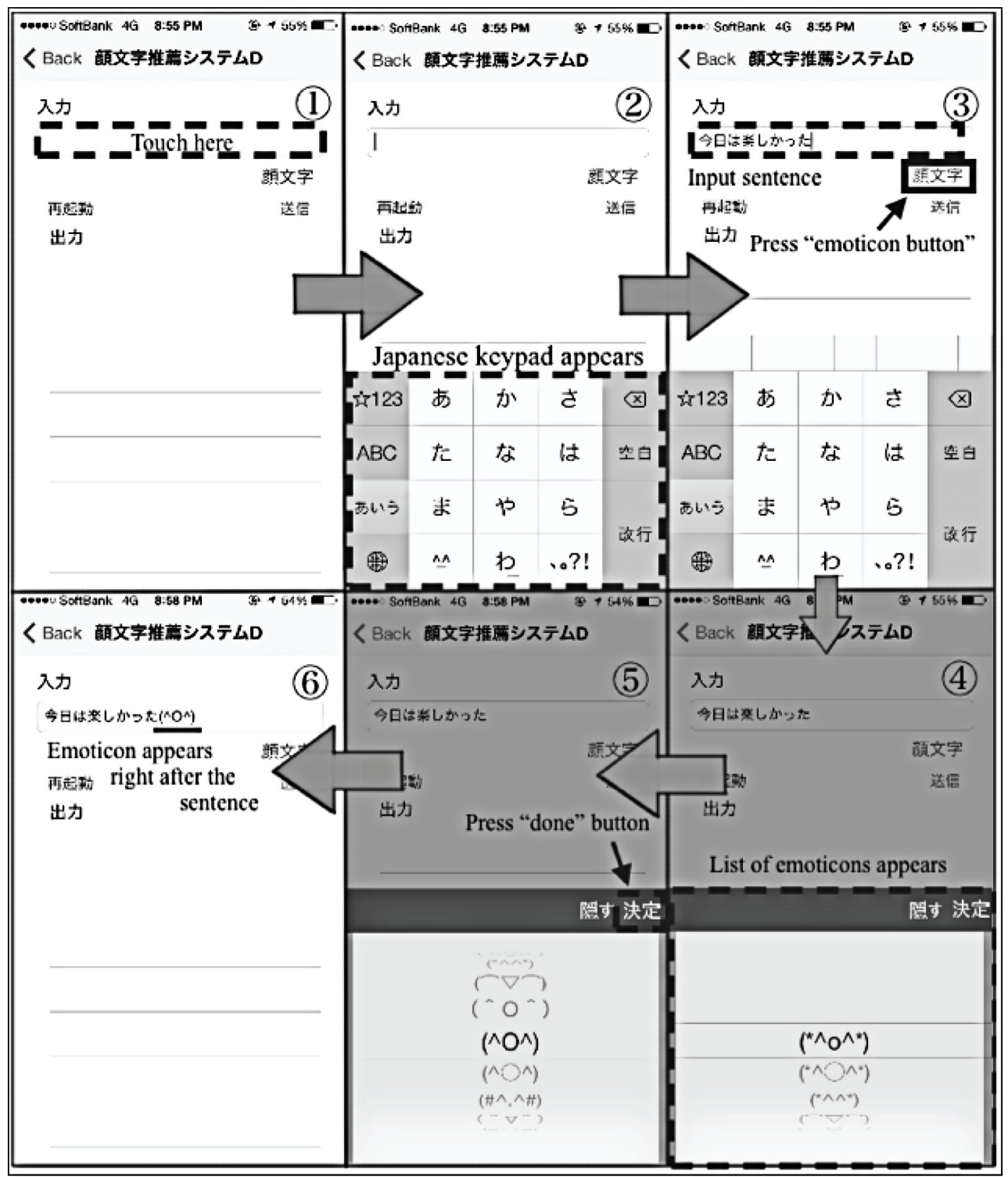

emoticons to users among the four systems. We can also confirm that this is true for both men ( 4.4 points) and women (5.6 points) from the same figures. The second best performance is the EBReS (overall: 6.9, men: 6.5, and women: 7.4), which scored a little lower than the HiBReS- $\varepsilon$. On the other hand, the HiBReS showed the highest value, which implies that users had to scroll down to find suitable emoticons for their input. Although the HiBReS performs the worst among the four systems, this method is still helpful when incorporated with the method of the EBReS, which we can see from the difference between the EBReS 
Table 4. Example of sentences shown to participants

\begin{tabular}{|l|l|l|l|}
\hline \multicolumn{4}{|c|}{ Example of Sentences Shown to Participants } \\
\hline \multicolumn{1}{|c|}{ Japanese Sentence } & \multicolumn{1}{|c|}{ Transliteration } & \multicolumn{1}{c|}{ Translation } & \multicolumn{1}{c|}{ Emotion } \\
\hline その漫画は好きだよ。 & $\begin{array}{l}\text { Sono manga ha suki } \\
\text { dayo. }\end{array}$ & I like this comic book. & $\begin{array}{l}\text { Liking/fondness (4.9) } \\
\text { (Positive) }\end{array}$ \\
\hline $\begin{array}{l}\text { それはちよつと } \\
\text { 恥ずかしい。 }\end{array}$ & Sore ha chotto hazukashi. & $\begin{array}{l}\text { This is a little } \\
\text { embarrassing. }\end{array}$ & Shyness (4.3) (Neutral) \\
\hline 怯えてしまう。 & Obiete shimau. & I am frightened. & Fear (4.8) (Negative) \\
\hline
\end{tabular}

Figure 7. Average rank of emoticons chosen by users with each system

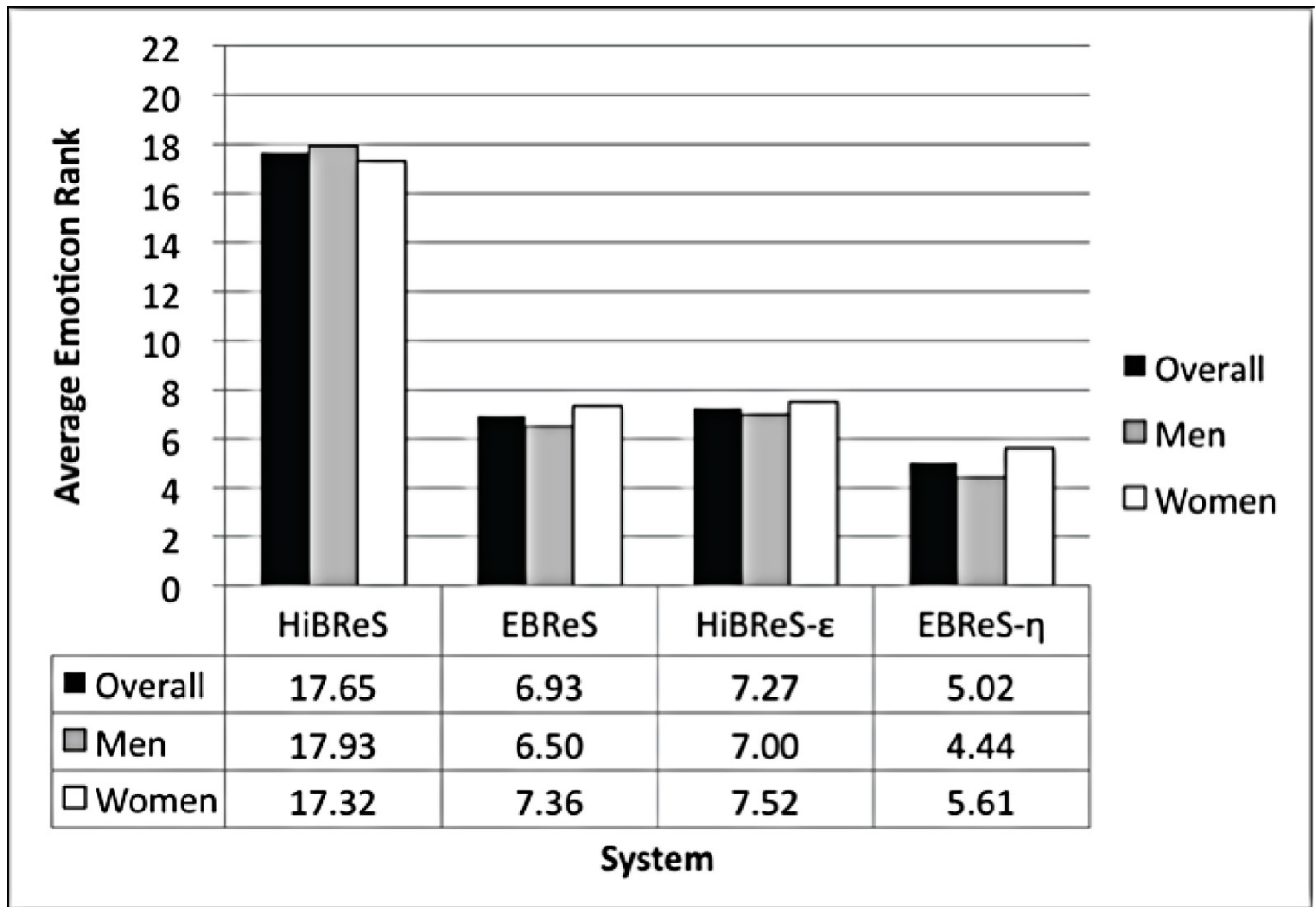

and the EBReS- $\eta$. From these results, we can assert that recommending emoticons based on emotional statements is helpful to users, and that an improved performance can be achieved when we apply users' past emoticon selection data to the emotion-based recommending method.

We broke down the overall results into positive (fondness/liking, joy/delight, and relief), neutral (excitement, shyness, and surprise/ amazement), and negative (anger, dislike, fear, and sadness/gloom) emotions (Figures 8 to 10). From these results, the EBReS- $\eta$ scored lowest for the negative emotions in both the results of men and women. We assume that this result comes from the lower number of negative emoticons (Figure 5; anger: 3 emoticons, fear: 2 emoticons, sadness/gloom: 10 emoticons, and dislike: 13 emoticons) in the database, so candidates for the negative contexts were limited. In contrast, there are a higher number of positive emoticons (Figure 5; joy/delight: 25 emoticons, fondness/liking: 22 emoticons, and relief: 22 emoticons), so when users input positive statements there are more candidates than negative ones. Also, we discovered that women gave higher scores than men in most 
Figure 8. Average rank of emoticons chosen by users with each system (Positive). Positive emotion types included in this data are "joy/delight", "fondness/liking", and "relief".

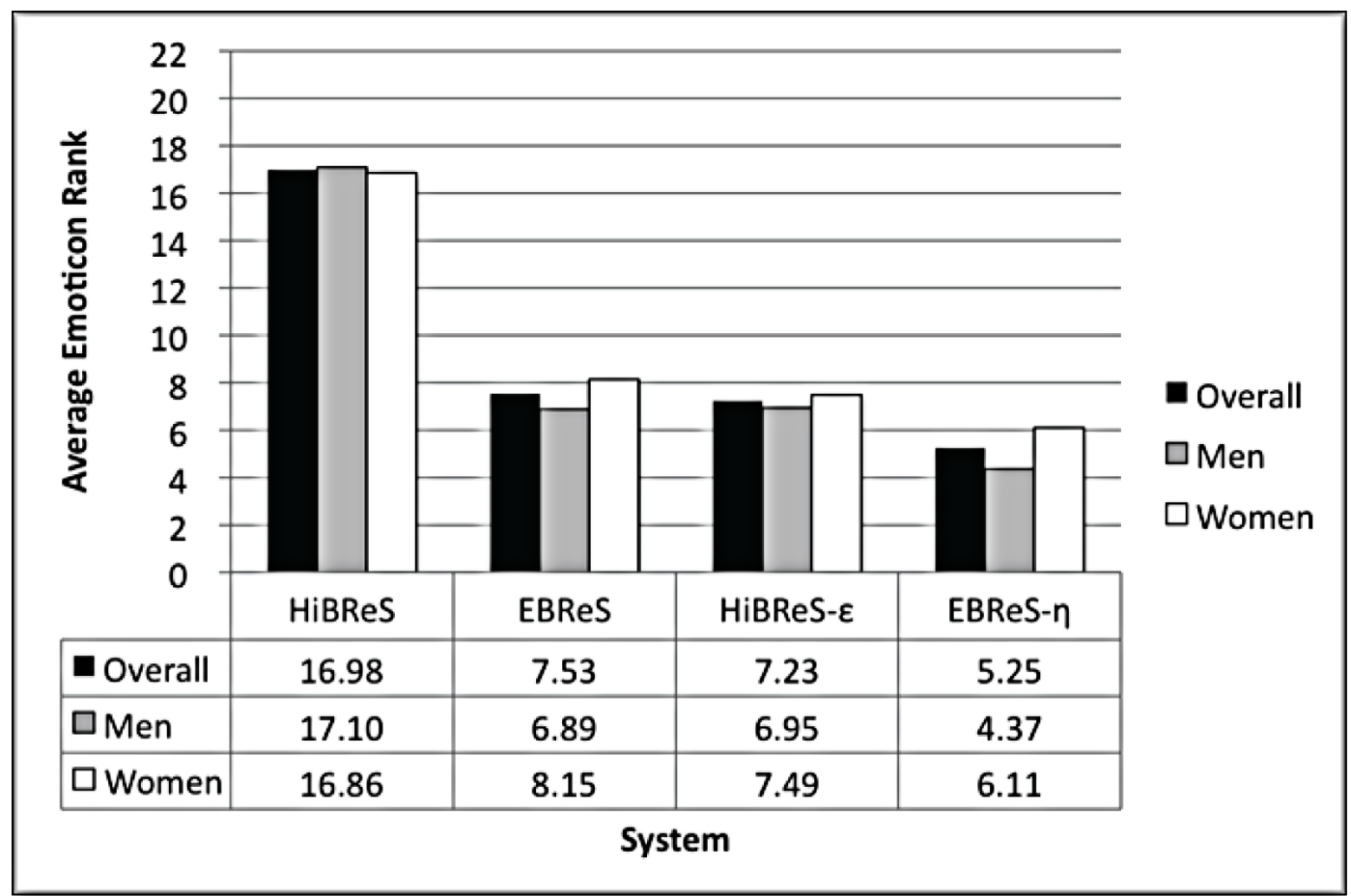

Figure 9. Average rank of emoticons chosen by users with each system (Neutral). Neutral emotion types included in this data are "excitement", "shyness", and "surprise/amazement".

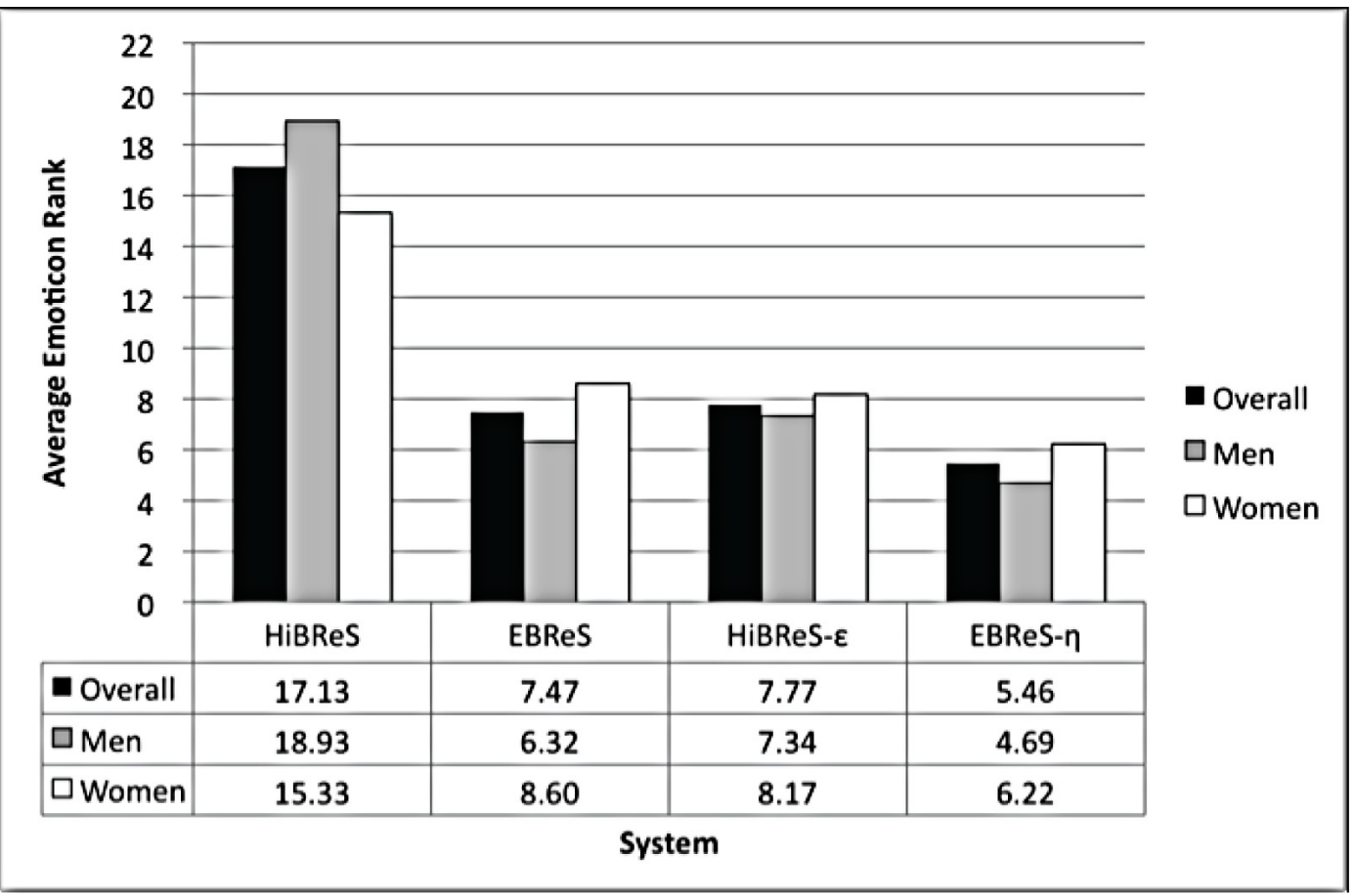


Figure 10. Average chosen emoticon rank using each system (Negative). Negative emotion types included in this data are "anger", "dislike", "fear", and "sadness/gloom".

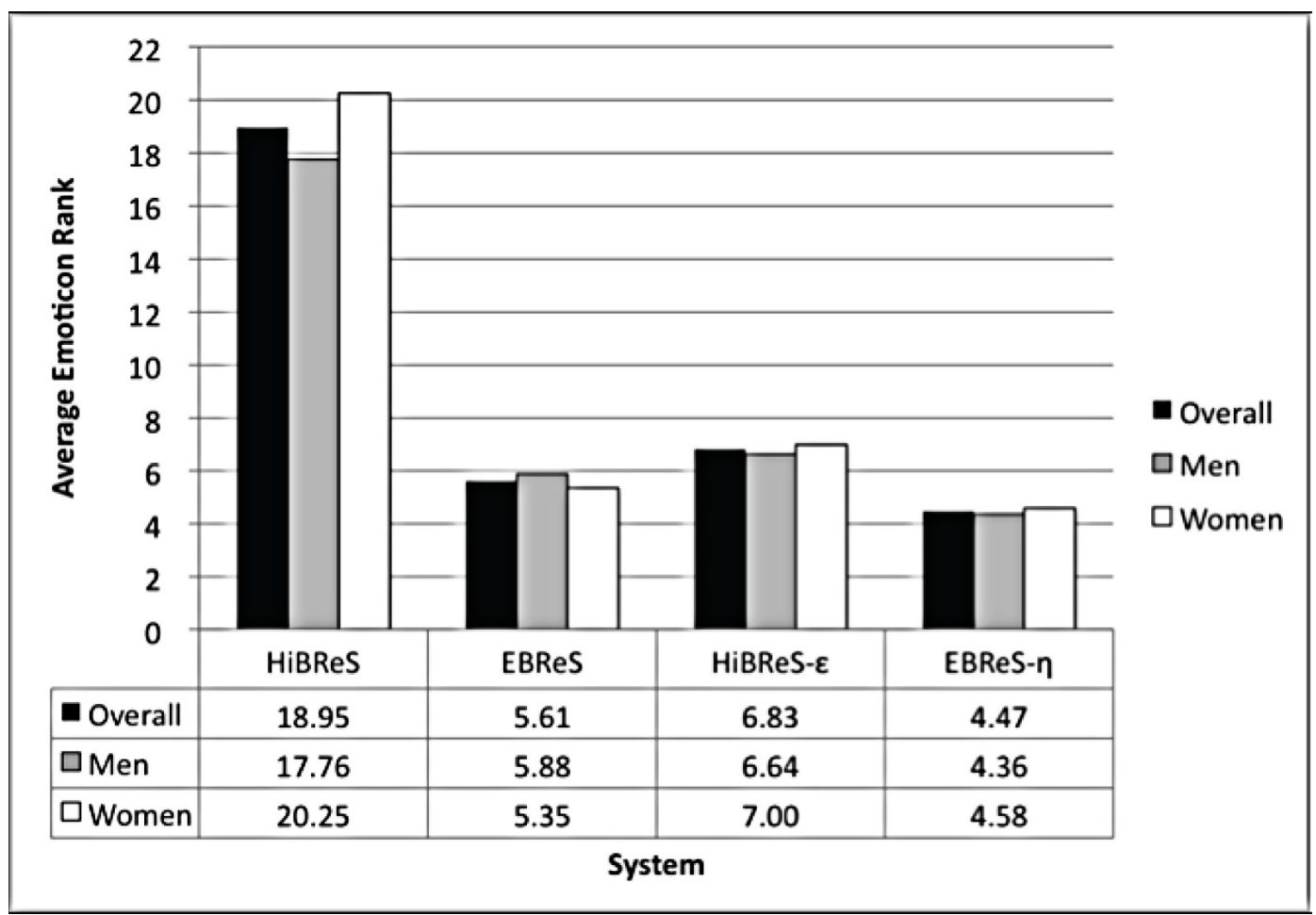

of the results. This may indicate that women tend to look at the list more thoroughly than men and choose different kinds of emoticons.

\section{Proportion of Emoticons Chosen by Users Among the Top Five from Each System}

We investigated the ratio of users choosing emoticons from among the top five from each system (Figure 11). In Figure 11, we can judge that the higher the value is, the better the system assists users to insert suitable emoticons into their statements easily. Figure 11 shows that the EBReS- $\eta$ scored the highest, at $73.0 \%$, among the four systems. This value represents an improvement of $43.5 \%$ from the HiBReS score. As previously mentioned, the Japanese iOS emoticon recommendation system utilizes the same recommendation method as the HiBReS, so we can assume that users are scrolling down the list repeatedly to find appropriate emoticons for their statements, which is not efficient. We were also able to determine that emotions detected from users' statements are extremely reliable when recommending emoticons (Figure 11, EBReS \& EBReS- $\eta$ ).

We broke down the overall results into positive, neutral, and negative in order to investigate more precisely. Figures 12 to 14 are the results of the proportion of emoticons chosen among the top five when users' statements were positive, neutral, and negative, respectively. We discovered that the proportion of the EBReS- $\eta$ from Figure 14 (negative) was relatively higher than others. Moreover, we can see a greater difference between the EBReS- $\eta$ and the HiBReS- $\varepsilon$ with negative than with positive and neutral. We assume that this result is due to the smaller number of negative emoticons in the database than emoticons that express positive or neutral emotions. We also discovered that the EBReS scored higher than the HiBReS- $\varepsilon$ in most of the results, but women using the HiBReS- $\varepsilon$ with neutral statements scored higher than the EBReS. This lower score of female users of the EBReS may indicate that there were only a few emoticons expressing "surprise/amazement", 
Figure 11. Proportion of emoticons chosen among the top five

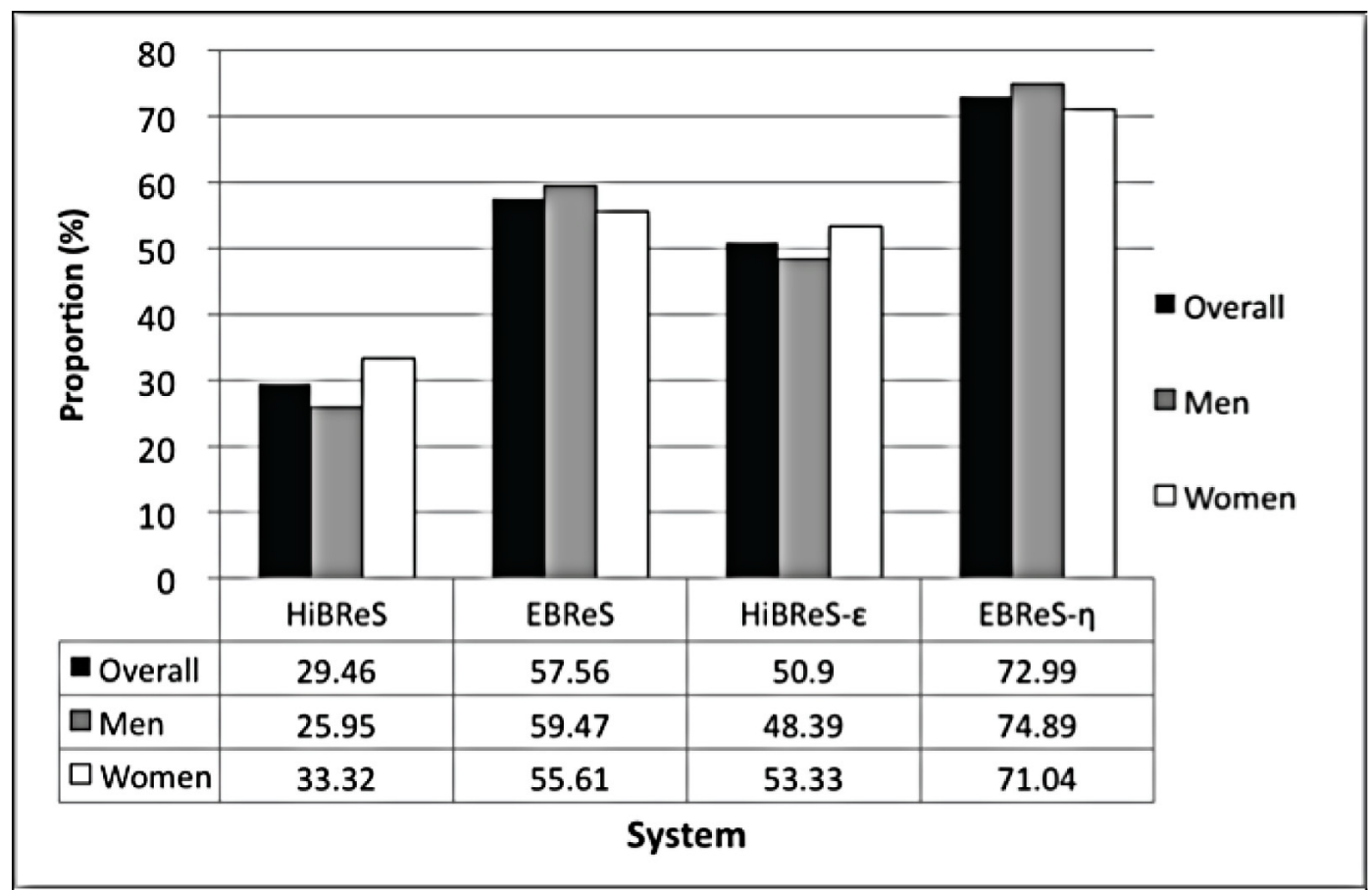

Figure 12. Proportion of emoticons chosen among the top five (Positive). Positive emotion types included in this data are "joy/delight", "fondness/liking", and "relief".

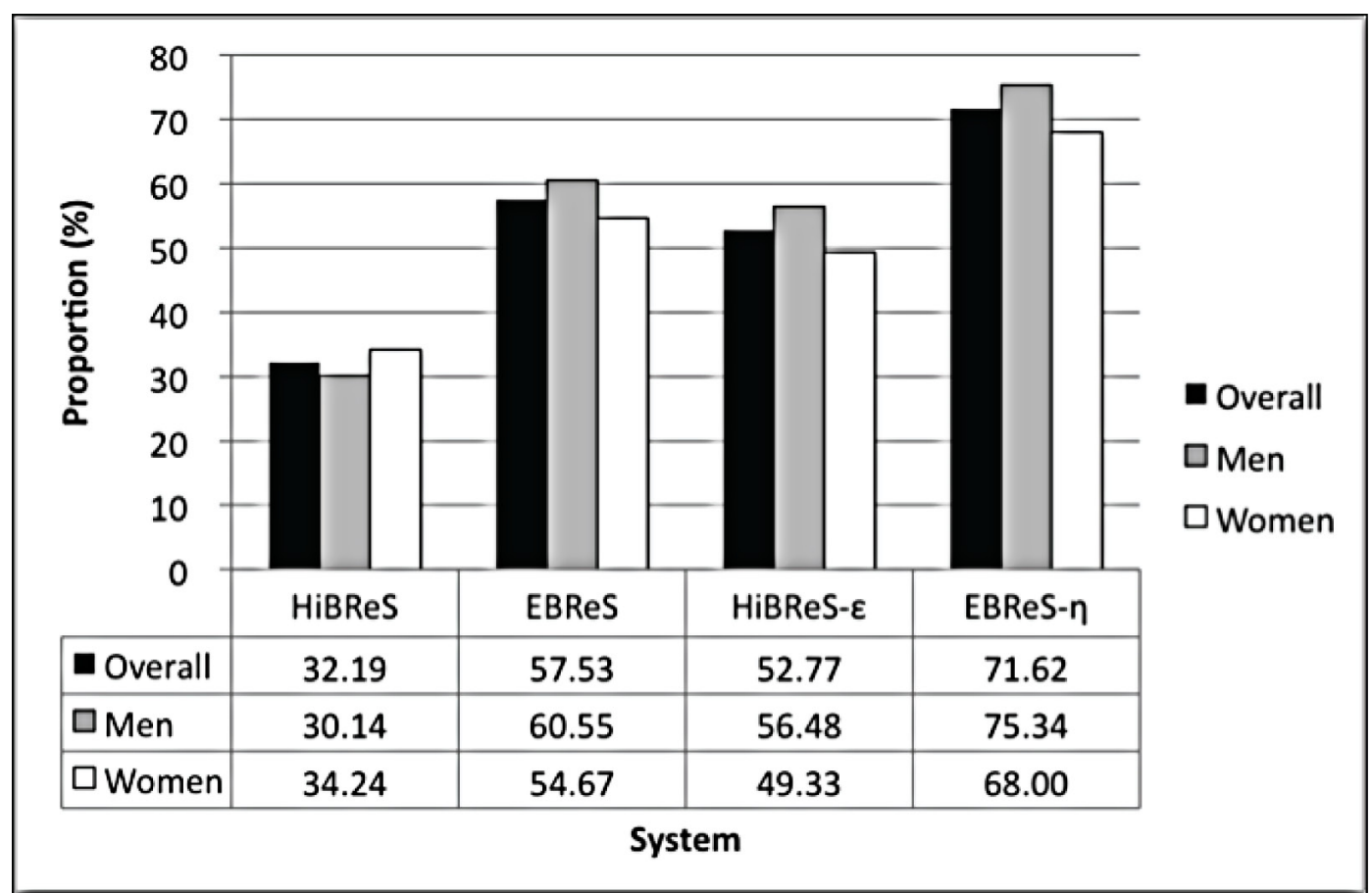


Figure 13. Proportion of emoticons chosen among the top five (Neutral). Neutral emotion types included in this data are "excitement", "shyness", and "surprise/amazement".

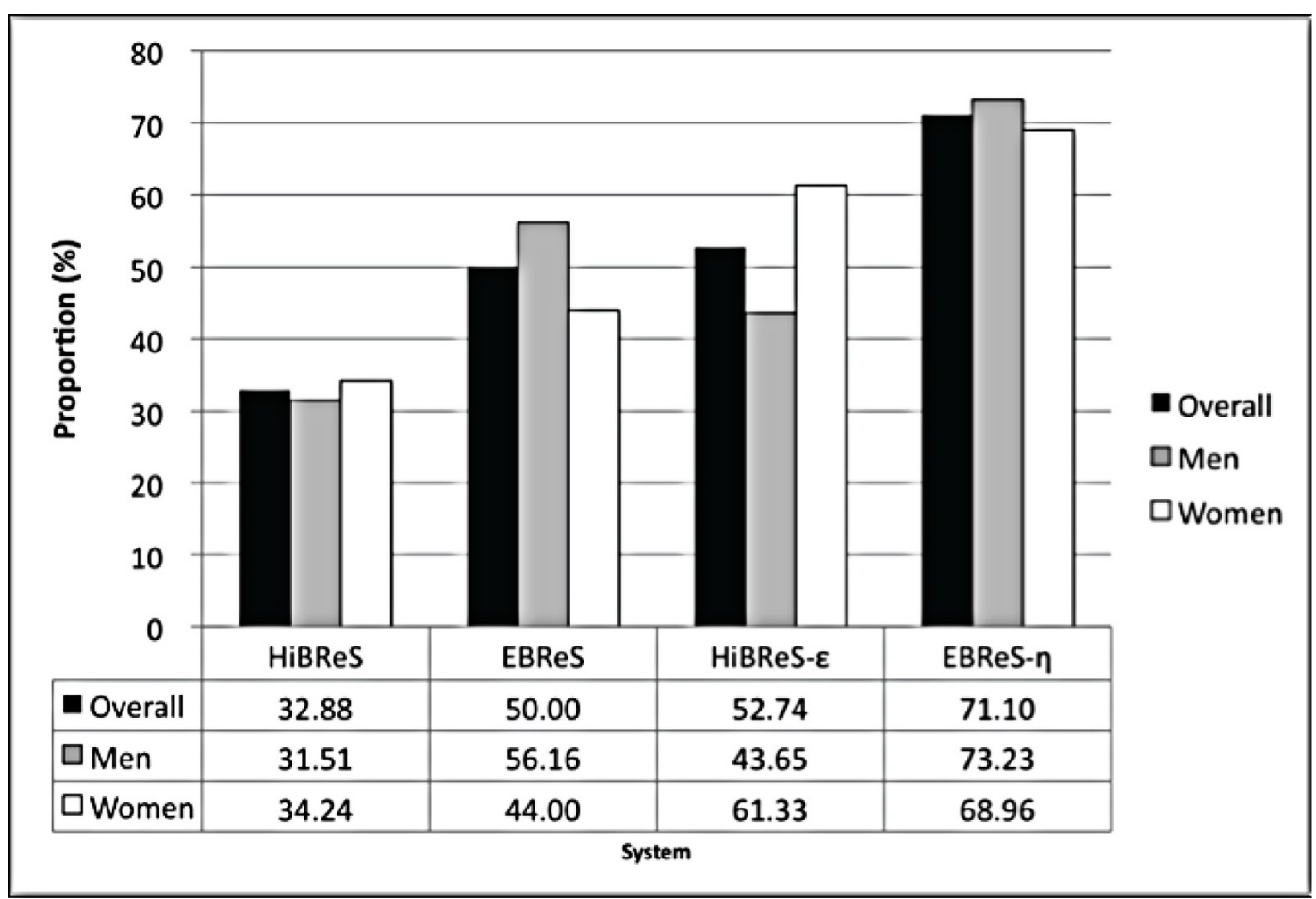

Figure 14. Proportion of emoticons chosen among the top five (Negative). Negative emotion types included in this data are "anger", "dislike", "fear", and "sadness/gloom".

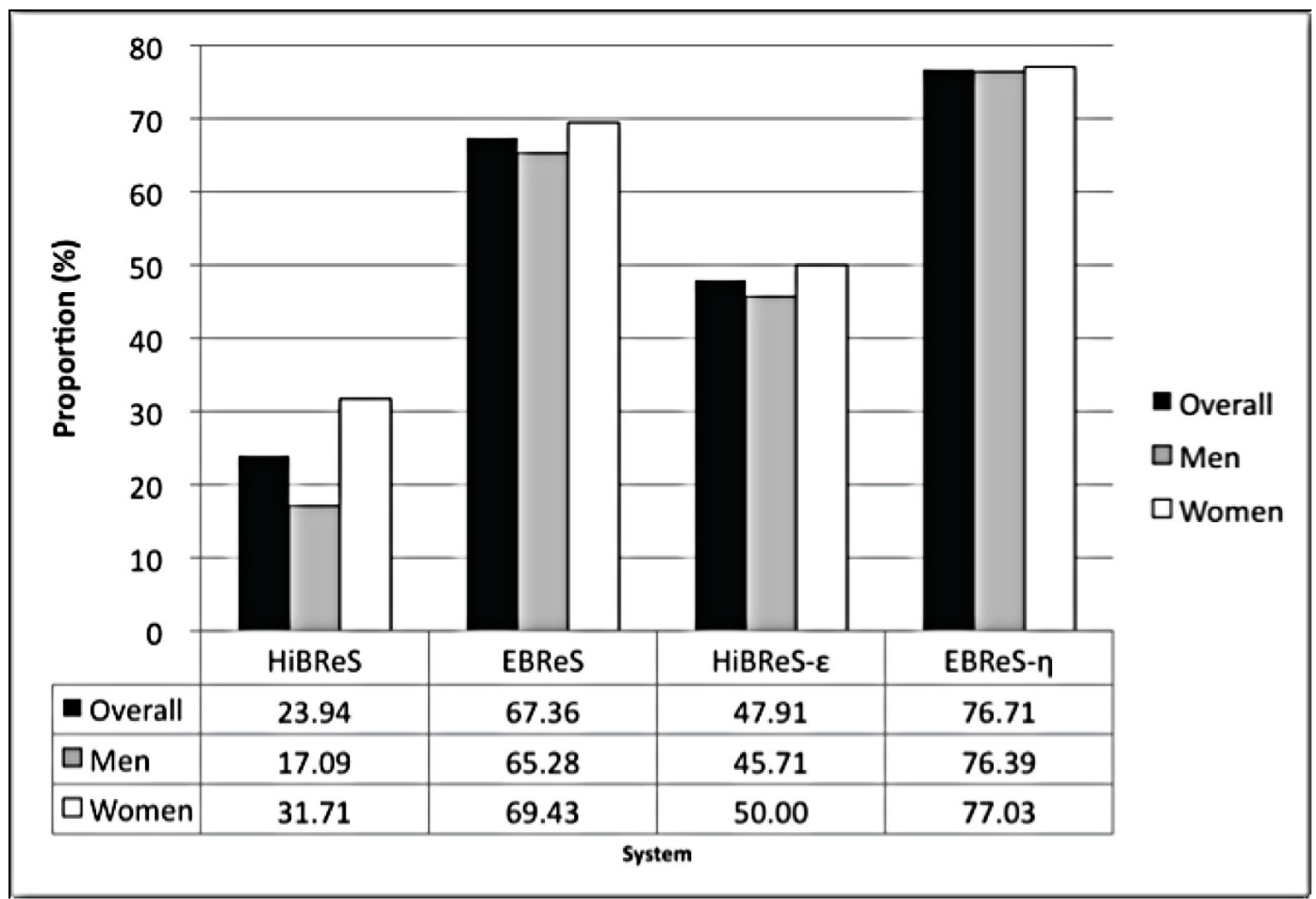


"excitement", and "shyness" for women in particular, so female users had to find emoticons that were ranked lower. However, the HiBReS- $\varepsilon$ recommends based on users' past selection, so once they have chosen suitable emoticons for neutral statements, these reflect their future choice of emoticons.

In order to obtain higher results, we believe that we should also adopt machine-learning techniques to learn the patterns of what kind of emoticons are frequently used with which words in a sentence, and recommend emoticons depending on these data. Moreover, by employing machine-learning techniques, we can make our system capable of recommending emoticons when there are no emotive words in the input.

\section{Participants' Perception using SD Scale}

We verified that the EBReS- $\eta$ performs the best by analyzing the rank of chosen emoticons, but this is not enough to fully confirm the EBReS$\eta$ 's superiority. Therefore, we examined participants' perceptions towards each of the four systems using the results from the SD scale. In Figure 15, numbers closer to one have strong impressions of the words on the left, whereas numbers closer to seven are better characterized by the words on the right. The average is shown under each system.

From Figure 15, we discovered that the EBReS- $\eta$ scored the highest among the four

Figure 15. Results of the SD scale

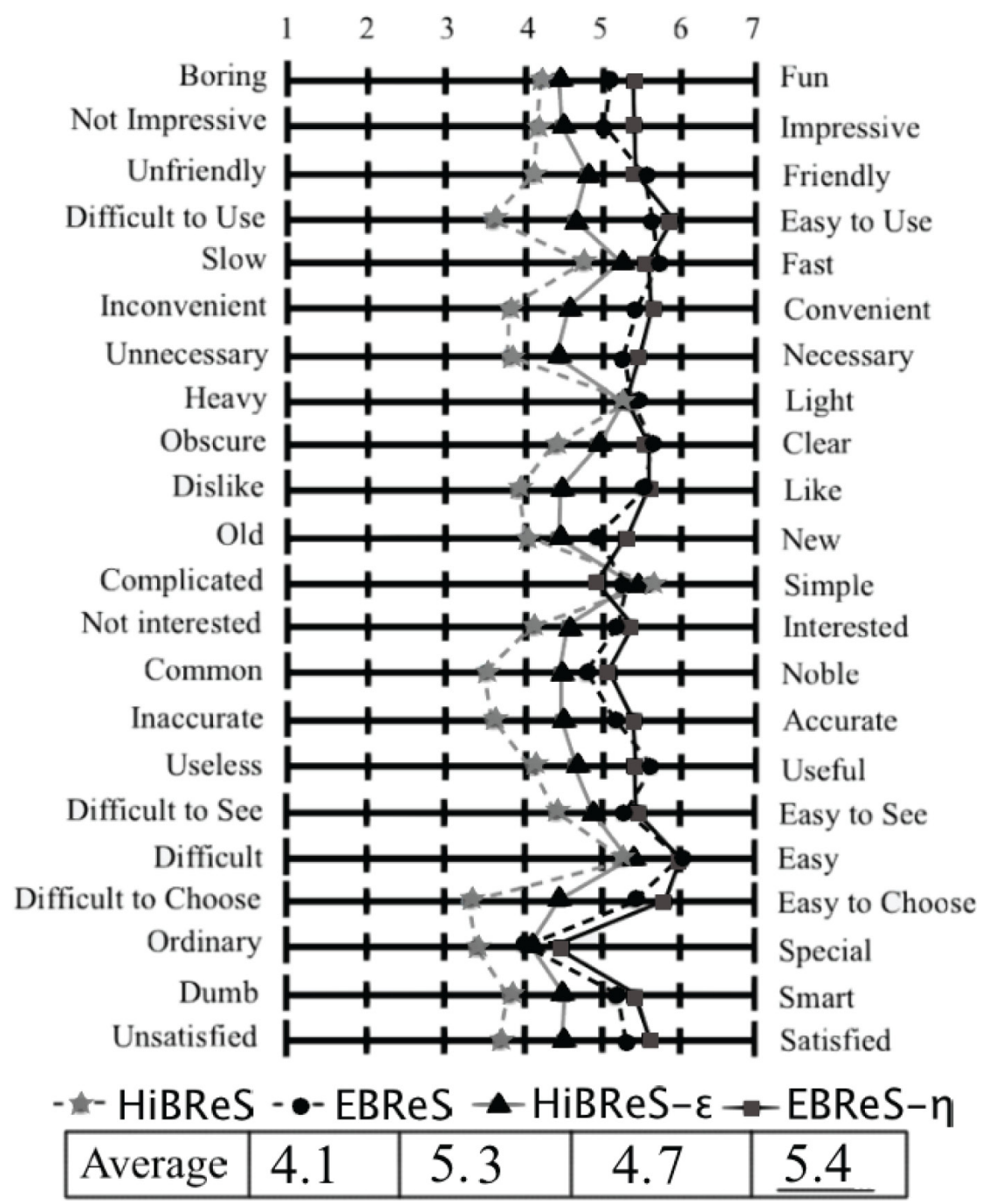


systems for 15 word pairs out of 22 word pairs. The overall average was 5.4 points (minimum is 1 , maximum is 7). The second highest was the EBReS, which was slightly lower than the EBReS- $\eta$. From this result, it is clear that recommending emoticons according to emotion types from user input is effective for users to choose emoticons easily. We also found that the EBReS- $\eta$ (4.9 points) and EBReS (5.4 points) scored lower than the HiBReS- $\varepsilon$ (5.5 points) and HiBReS (5.6 points) for the word pair "complicated - simple". We assumed that most participants rated this by considering the process of the system recommending emoticons to them.

\section{CONCLUSION}

In this paper, we presented three emoticon recommendation systems (EBReS, EBReS- $\eta$, and HiBReS- $\varepsilon$ ) based on users' past emoticon selection and emotional statements. The main procedures of these emoticon recommendation systems share the same process of analyzing emotions from user-entered sentences by using the affect analysis system ML-Ask, but differ in their methods of reordering the emoticon database and recommending appropriate emoticons to users. The emoticon database was originally created by conducting a questionnaire towards 60 participants to rate 59 emoticons in terms of how well they express each of 10 emotions, and calculating and registering the average points in the database.

The results of our experiment showed that approximately $73.0 \%, 57.6 \%$, and $50.9 \%$ of chosen emoticons were among the top five recommendations by the EBReS- $\eta$, EBReS, and HiBReS- $\varepsilon$, respectively. On the other hand, the current system used in the Japanese iPhone keypad only achieved $29.5 \%$ in the same experiment. From the overall experiment results, we have confirmed that emotion plays a major role when recommending appropriate emoticons to users.
Our future work will be to develop a method for learning which kinds of emoticons are preferred for which words in the sentence, so that our system will also work with sentences with no emotive words. Moreover, expansion of the emoticon database is also required. More emoticons in the database will be helpful for discovering the types of symbols that articulate each emotion type, and in order to create a system to generate emoticons suitable to the input.

\section{REFERENCES}

Ashcraft. B. (2012). The latest Japanese emoticons arefreaky, yet wonderful. KOTAKU. Retrieved January 6, 2014, from http://kotaku.com/5941811/thelatest-japanese-emoticons-are-freaky-yet-wonderful

Derks, D., Bos, A. E. R., \& Grumbkow, J. B. (2008). Emoticons in computer-mediated communication: Social motives and social context. Cyberpsychology \& Behavior, 11(1), 99-101. doi:10.1089/ cpb.2007.9926 PMID:18275321

Dilger, D. E. (2010). iOS 4 includes emoji input on Japanese keyboard. Apple Insider. Retrieved January 7, 2014, from http://appleinsider.com/ articles/10/06/25/ios 4 includes emoji input on japanese_keyboard

Emoticons. (2013), techdictionary.com. Retrieved November 26,2013, from http://www.techdictionary. com/emoticon_cont3.html

Emura, Y., \& Seki, Y. (2012). Facemark recommendation based on emotion, communication, and motion type estimation in text [in Japanese]. IPSJ Sig Notes, 85, 1-7.

Fridlund, A. J. (1994). Human facial expression: An evolutionary view. San Diego, CA: Academic Press.

Gajadhar, J., \& Green, J. (2005). The importance of nonverbal elements in online chat. EDUCAUSE Quarterly, 28(4), 2.

Ip, A. (2002). The impact of emoticons on affect interpretation in instant messaging. Retrieved from http://www.amysmile.com/doc/emoticon_paper.pdf

Jibril, T. A., \& Abdullah, M. H. (2013). Relevance of emoticons in computer-mediated communication contexts: An overview. Asian Social Science, 9(4), 201-207. doi:10.5539/ass.v9n4p201 
Kato, S., Kato, Y., \& Scott, D. (2009). Relationships between emotional states and emoticons in mobile phone email communication in Japan. International Journal on E-Learning, 8(3), 385-401.

Kawakami, M. (2008). The database of 31 Japanese emoticon with their emotions and emphasis [in Japanese]. The Human Science Research Bulletin of Osaka Shoin Women's University, 7, 67-82.

Lo, S. K. (2008). The nonverbal communication functions of emoticons in computer-mediated communication. Cyberpsychology \& Behavior, 11(5), 595-597. doi:10.1089/cpb.2007.0132 PMID:18817486

Mehrabian, A. (1971). Silent messages (1st ed.). Belmont, CA: Wadsworth.

Nakamura, A. (1993). Dictionary of emotive expressions (Kanjyo Hyogen Jiten). Tokyo, Japan: Tokyodo Publishing. (in Japanese)

Osgood, C. E., Suci, G. J., \& Tannenbaum, P. H. (1957). The measurement of meaning. Urbana, IL: University of Illinois Press.

Park, J., Barash, V., Fink, C., \& Cha, M. (2013). Emoticon style: Interpreting differences in emoticons across cultures. In Proceedings of the Seventh International AAAI Conference on Weblogs and Social Media (pp. 466-475).

Pollack, A. (1996). Happyin the East $\left({ }^{\wedge} \_\right.$^) or smiling in the West:). The New York Times, p. D5.

Ptaszynski, M. (2012). Robust NLP for real-world data: 1. Emoticon processing - How to non-trivially make a computer understand trivial expressions - [in Japanese]. Information Processing Society of Japan, 53(3), 204-210.

Ptaszynski, M., Dybala, P., Rzepka, R., \& Araki, K. (2008). Effective analysis of emotiveness in utterances based on features of lexical and non-lexical layer of speech. In Proceedings of the Fourteenth Annual Meeting of the Association for Natural Language Processing (pp. 171-174).
Ptaszynski, M., Maciejewski, J., Dybala, P., Rzepka, R., \& Araki, K. (2010). CAO: A fully automatic emoticon analysis system based on theory of kinesics. IEEE Transaction on Affective Computing, 1, 46-59. doi:10.1109/T-AFFC.2010.3

Robb, T. (2003). e-Mail emoticons in a cultural context. In Dorothy Zemach's From A to Z column, "uve got mail," essential teacher (pp. 19-21).

The Facemark Dictionary. (2004). The facemark dictionary. Retrieved November, 26, 2013 from http://www.kaomoji.sakura.ne.jp/

Tossell, C. C., Kortum, P., Shepard, C., Barg-Walkow, L. H., Rahmati, A., \& Zhong, L. (2012). A longitudinal study of emoticon use in text messaging from smartphones. Computers in Human Behavior, 28, 659-663. doi:10.1016/j.chb.2011.11.012

Urabe, Y. (2013). Creation and evaluation of emoticon recommendation system using emoticons categorized by emotions (in Japanese) (Unpublished bachelor thesis). Department of Engineering, Hokkaido University, Sapporo - Japan.

Urabe, Y., Rzepka, R., \& Araki, K. (2013). Emoticon recommendation for Japanese computer-mediated communication. In Proceedings of 2013 IEEE Seventh International Conference on Semantic Computing (pp. 25-31).

Walther, J. B., \& D'addario, K. P. (2001). The impacts of emoticons on message interpretation in computer-mediated communication. Social Science Computer Review, 19(3), 324-347. doi:10.1177/089443930101900307

Wei, A. C. Y. (2012). Emoticons and the non-verbal communication: With reference to Facebook(Unpublished master's thesis). Department of Media Studies, Christ University, Bangalore - India.

Wolf, A. (2000). Emotional expression online: Gender differences in emoticon use. $C y$ berpsychology \& Behavior, 3(5), 827-833. doi:10.1089/10949310050191809

Yuki Urabe was born in Toyota City, Japan, and received B.E. from Hokkaido University University, Japan in 2013. Currently, she is a master student at the Graduate School of Information Science and Technology, Hokkaido University. Her research interests are Natural Language Processing, Sentiment Analysis, and Text Mining. 
Rafal Rzepka was born in Sczecin, Poland, and received M.A. from Adam Mickiewicz University in Poznan, Poland in 1999 and Ph.D. from Hokkaido University, Japan in 2004. Currently he is an assistant professor at the Graduate School of Information Science and Technology, Hokkaido University. His research interests are Common Sense Acquisition, Affective Processing and Machine Ethics. He is a member of AAAI, ACL, IPSJ, IEICE, JCSS, and ANLP.

Kenji Araki received his B.E. and Ph.D. degrees from Hokkaido University in 1982 and 1988. He was an associate professor there from 1991 to 1998, and a professor in 1998 at Hokkai-Gakuen University. He then joined Hokkaido University as an associate professor. He is currently a professor at the Graduate School of Information Science and Technology. His research interests include natural language processing, morphological analysis, machine translation, and speech dialogue processing. He is a member of ACL, IEEE, AAAI, IPSJ, and NLP. 